DOI: $10.19195 / 0137-1169.38 .5$

\title{
Vokalquantitätsverschiebungen in ausgewählten Dorfwillküren aus dem 17. und 18. Jh. Eine graphematisch-phonematische Studie
}

\section{Einleitendes und Zielsetzung}

Die Vokalquantität als Lautlänge von gesprochenen Vokalen gehört - neben der Vokalqualität - zu den Begriffen, die im Begriffssystem und im wissenschaftlichen Instrumentarium der Sprachgeschichte und der Sprachgeschichtsuntersuchung tief verwurzelt sind (mehr dazu Szulc 1987:124-125, Szczepaniak 2007:60, $233-$ 234, 240). Beim Gebrauch dieser Begriffe kommt jedoch sofort die Annahme auf, dass es sich hier vordergründig um die gesprochene Sprache handelt. Im Falle der Sprachgeschichte hat man es eher selten mit der gesprochenen Sprache zu tun, weil die Untersuchungsbasen für die Sprachhistoriker häufig - wenn nicht immer - die Schriftdenkmäler aus längst verklungenen Epochen sind. Daraus resultiert wiederum das Problem der Äquivalenz zwischen der Rede und der Schrift, die nur selten vorkommt und nur in solchen Sprachen vorhanden ist, die keine lange Schreibtradition besitzen. ,[...] jeder Schreibende [arbeitet] noch selbstständig mit an der Schöpfung der Orthographie [...], indem zwar ungefähr feststeht, welches Zeichen für jeden einzelnen Laut zu wählen ist, aber nicht, wie das Wort als Ganzes zu schreiben ist, so dass es der Schreiber immer erst, so gut es angehen will, in seine Elemente zerlegen und die diesen Elementen entsprechenden Buchstaben zusammensetzen muss“ (Paul 1968:380-381).

Normalerweise gibt es also keine 1:1-Entsprechung zwischen der phonischen und der graphischen Ebene der Sprache, worauf schon mehrmals in der langen Kette der Sprachwissenschaftsgeschichte von unterschiedlichen Sprach- 
forschern hingewiesen wurde. Auf das trügerische Wesen der Schrift wurde die Aufmerksamkeit zuerst von den Junggrammatikern gerichtet (vgl. Paveau/Sarfati 2009:38), für die die phonische Sprachebene eine autonome und zugleich die wichtigste Forschungssprachschicht war. Einen solchen Standpunkt vertrat ebenfalls Jan Baudouin de Courtenay (1845-1929), der sich für eine gewisse Eigenständigkeit der Schrift als Möglichkeit der Aufbewahrung und der Weitergabe von menschlichen Ideen und Errungenschaften der Zivilisationsentwicklung zwischen den Generationen aussprach. Somit erkannte er die gesprochene Sprache als Mittel der hic-et-nunc-Kommunikation an (vgl. Baudouin de Courtenay 1974:52). Diesem Gesichtspunkt stand auch Ferdinand de Saussure (1857-1913) nahe, der die Rede und die Schrift als zwei voneinander unabhängige Systeme auffasste und behauptete, dass das Geschriebene nur als Abbild des Gesprochenen zu betrachten ist. „Der Gegenstand der sprachwissenschaftlichen Forschung soll aber ausschließlich die gesprochene Sprache sein. Das Geschriebene, das sich das Recht wichtiger als das Gesprochene zu sein, anmaße, müsse nur als das Abbild des Gesprochenen betrachtet werden. Die Tatsache, dass wir der geschriebenen Sprache mehr Relevanz zusprechen, erklärt de Saussure mithilfe der Illusion, die unsere Wahrnehmung der Sprache begleitet, z.B. die gesprochene Sprache unterliege schnelleren Veränderungen, wenn sie keine Schrift besitze. [...] Die gesprochene Sprache besitze also eine von der Schrift unabhängige Tradition, was wir nicht zu bemerken imstande sind“" (Owsiński 2017:19-20).

Zwar gibt es dieses Problem in der Glossematik nicht, weil die Kopenhagener Linguisten beide Systeme für zwei unabhängig koexistierende Sprachrealisierungsformen hielten (vgl. Uldall 1944:14-16, Penttilä 2011:5), aber bereits Leonard Bloomfield (1887-1949) sprach der Rede, die von der Schrift lediglich nachgeahmt wird und eine ganz andere Struktur besitzt, besonders Gewicht zu: „Writing is not a language, but merely a way of recording language by means of visible marks. In some countries, such as China, Egypt, and Mesopotamia writing was practiced thousands of years ago, but to most of languages that are spoken today it has been applied either in relatively recent times or not at all. Moreover, until the days of printing, literacy was confirmed to a very few people. All languages were spoken through nearly all of their history by the people who did not read or write; the languages of such peoples are just as stable, regular and rich as the languages of the literate nations. A language is the same no matter what system of writing may be used to record it, just as a person is the same no matter how you take his picture. [...] In order to study writing, we must know something about language, but the reverse is not true" (Bloomfield 1933:21).

Das Hauptziel des vorliegenden Beitrags ist also der Versuch der Ermittlung, ob und inwieweit die Schrift die subtilsten Eigentümlichkeiten des Gesprochenen, denen sicherlich die Länge und die Kürze des Vokals angehören, notiert und widerspiegelt, wobei mitberücksichtigt werden soll, dass das Untersuchungskorpus aus dem 17. und 18. Jh. - also aus der (früh-)neuhochdeutschen (fnhd.) Periode 
laut der Sprachgeschichtsperiodisierung von Wilhelm Scherer (1841-1886) stammt, als es noch keine festen orthographischen Regeln gab. Da das Fnhd. aber als ein hypothetisches Gefüge angesehen wird, kann man sich auch auf das frühere künstliche und idealisierte Mittelhochdeutsch beziehen, das doch als solch ein Entwicklungsstadium gilt, das samt seinen häufig als fnhd. bezeichneten Neuerungen dem heutigen Deutsch weithin das gegenwärtige Antlitz angedeihen ließ. Aus diesem Grunde werden die in den zu untersuchenden Texten aus dem 17. und 18. Jh. schriftlich fixierten Lautwandelsformen in Beziehung mit dem mhd. Lautinventar gesetzt. Dabei muss jedoch die folgende Feststellung von Hermann Paul (1846-1921) als entscheidend betrachtet werden: „Es ist wichtig für jeden Sprachforscher niemals aus den Augen zu verlieren, dass das Geschriebene nicht die Sprache selbst ist, dass die in Schrift umgesetzte Sprache immer erst einer Rückumsetzung bedarf. [...] Diese Rückumsetzung ist nur in unvollkommener Weise möglich [...]; soweit sie aber überhaupt möglich ist, ist sie eine Kunst, die gelernt sein will, wobei die unbefangene Beobachtung des Verhältnisses von Schrift und Aussprache, wie es gegenwärtig bei den verschiedenen Völkern besteht, große Dienste leistet“ (Paul 1968:373).

Das geschriebene Gegenwartsdeutsche verfügt jedoch über eine ganze Reihe von feinen konventionalisierten Möglichkeiten, die dem Leser die Vokallänge bzw. -kürze zu signalisieren imstande sind. Während die kurzen Vokale meistens in den Nebensilben auftreten ${ }^{1}$, können die Hauptsilbenvokale einige Bezeichnungen haben, dank denen ihre Quantität zum Ausdruck gebracht wird.

$\mathrm{Zu}$ den Längenmarkern der betonten Silben in der geschriebenen Sprache gehören u.a.: die Vokallautverdoppelung $\left(\mathrm{V}_{1}{ }^{2}+\mathrm{V}_{1}+\mathrm{K}^{3}\right.$, z.B. Aas [a:s]; $\mathrm{K}+\mathrm{V}_{1}+\mathrm{V}_{1}+\mathrm{K}$, z.B. Saal ['za:l]; $\mathrm{K}+\mathrm{K}+\mathrm{V}_{1}+\mathrm{V}_{1}$, z.B. Klee [kle:]), das postvokalische $<\mathrm{h}>$ (Dehnungs-h) $(\mathrm{V}+<\mathrm{h}>-$, z.B. ahnen ['a:nən]; $-\mathrm{V}+<\mathrm{h}>-$, z.B. dehnen ['de:nən]; $-\mathrm{V}+<\mathrm{h}>$, z.B. Kuh [ku:]) oder die Markierung des langen /i:/ mithilfe des nachgestellten $<\mathrm{e}>\left(<-\mathrm{i}+\mathrm{e}->\right.$ z.B. Liebe ['li:bə], verlieren [fe $\varepsilon^{\mathrm{e}}$ 'li:rən]).

Die Vokalkürze in der Schrift kann u.a. durch folgende Möglichkeiten angedeutet werden: die Verdoppelung eines konsonantischen Graphems nach einem vorangehenden vokalischen Graphem $\left(-\mathrm{V}+\mathrm{K}_{1}+\mathrm{K}_{1^{-}}\right.$, z.B. Mette ['metə], Wille ['vila]) oder das Konsonantencluster nach einem vokalischen Graphem $\left(-\mathrm{V}+\mathrm{K}_{1}+\mathrm{K}_{2^{-}}{ }^{4}\right.$, z.B. Angst [ayst], Mensch [menf]).

1 In Zusammensetzungen oder einigen Präfixen und Suffixen erscheinen aber die Langvokale, u.a. Lebewohl ['le:bəvo:1], Vortrag ['fo:"tra:k], sonderbar ['sond"ba: ${ }^{\mathrm{p}}$ ], Reichtum ['raiçtu:m], was darauf zurückzuführen ist, dass die sog. schweren Ableitungssilben ursprünglich als separate Lexeme mit ihrem eigenen Akzent funktionierten, die nicht selten in Zusammensetzungen vorkamen. Dieser selbstständige Akzent verwandelte sich mit der Zeit in den bis heute existierenden Nebenton, dank dem die Länge des Vokals geschützt werden konnte (vgl. Mettke 1970:74).

2 V. - Vokal.

${ }^{3}$ K. - Konsonant.

${ }^{4}$ Manche Konsonantenhäufungen markieren aber ausnahmslos einen langen vorangehenden Vokal,

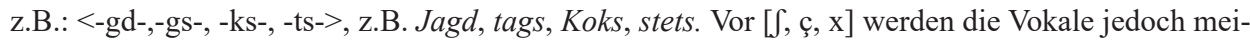


Die oben skizzierten orthographischen Feinheiten wurden aber verhältnismäßig spät ausgearbeitet und dürfen keinesfalls direkt auf die Schreibweise aus der Vergangenheit übertragen werden, als einheitliche Rechtschreibungsregeln noch keine Selbstverständlichkeit waren: Die ersten Anweisungen zur Orthographie begannen schließlich erst im 15. und 16. Jh. zu erscheinen und hatten eher keinen überregionalen Charakter: Niklas von Wyle (um 1410-1479), Heinrich Steinhöwel (1410/11-1479), Hans Nythart (die erste Hälfte des 15. Jhs.-1490) sowie Friedrich Riederer (um 1450-um 1510) (vgl. Höchli 1981:22). Die geschriebene Sprache sowohl in den Stadt- als auch in den Dorfkanzleien im 17. und 18. Jh. war vielmehr von anderen Faktoren abhängig und deswegen , ,[...] ein Ergebnis der Auseinandersetzung der Mundart des Schreibers, jener Schriftsprache, die dieser erlernt hat, des Usus der Kanzlei, der mehrmals abgeschriebenen Vorlage und - was oft vergessen wird - des Usus beim Empfänger" (Chromik 2010:27).

\section{Untersuchungskorpora}

Die Untersuchungsbasis bilden zwei Dorfwillküren:

1. Wilkühr des Closters Marien Paradeiss Cartheuser Ordens (17. Jh.) aus Karthaus (poln. Kartuzy) im Karthäuser Kreis in der polnischen Woiwodschaft Pommern (poln. województwo pomorskie);

2. Willkür von Dörfern - Kosowo und Chrystkowo (18. Jh.), die im Kreis Schwetz (poln. Świecie) in der Woiwodschaft Kujawien-Pommern (poln. województwo kujawsko-pomorskie) gelegen sind.

Die beiden Sammlungen von Rechtsvorschriften, die in den heutzutage polnischen Städten und - ab dem 16. Jh. - auch Dörfern Markt-, Zunft-, Sicherheits- und Gesellschaftsordnungsangelegenheiten regelten, können den Urkunden zugerechnet werden, die in den von der Ostsiedlung betroffenen Gebieten entstanden. „Die deutsche Ostkolonisation lässt sich [...] auf keinen Fall nur als eine aggressive Expansion des Deutschtums betrachten. [...] Die deutschen Kolonisten brachten [...] Fortschritt ins Land, und zwar in allen möglichen Bereichen des Lebens (Innenpolitik, Rechtswesen, Wirtschaft und Alltagsleben). Die Ostkolonisation hat also die wirtschaftliche und gesellschaftlich-kulturelle Entwicklung dieser Gebiete beschleunigt. Die Bauern hofften auf Befreiung von drückenden feudalen Lasten, die Handwerker auf Freiheit in den neuen Städten sowie günstigere Produktions- und Absatzmöglichkeiten. Alle suchten nach einer gesicherten Existenz" (Grabarek 2004:511-512).

stens kurz ausgesprochen: da ihre graphemischen Zeichen Konsonantenhäufungen darstellen: $<$ sch, ch, ch>, z.B. Asche [a jə], ich [iç], Krach [krax]. 
Das erste Dokument stammt aus dem 1938 von der Juristischen Kommission der Polnischen Akademie der Gelehrsamkeit (poln. Komisja Prawnicza Polskiej Akademii Umiejętności) herausgegebenen Band unter dem Titel: Archiwum Komisji Prawniczej / Tom XI, während sich das Manuskript der zweiten, auf zehn Blättern eines Papierheftes niedergeschriebenen Willkür in der Danziger Bibliothek der Polnischen Akademie der Wissenschaften befindet (vgl. Targowski 2013:14). Zwar sind beide Untersuchungskorpora nur ein kleiner Ausschnitt aus dem breiten Reichtum von Archivalien, in denen das Deutsche niedergeschrieben wurde, aber sie gewähren dennoch einen interessanten und mehr oder weniger detaillierten Einblick in die Richtung der Sprachentwicklung. Anhand dieser Texte, die im hundertjährigen Abstand entstanden sind, wird es überdies ermöglicht, die Gebrauchsmöglichkeiten bestimmter schriftlicher Lösungen der Markierung von den Vokalquantitätsverschiebungen sowie die Richtung dieses Vorgangs innerhalb von Jahrzehnten zu beobachten.

\section{Lautdauerveränderungen im Lichte der Schreibgewohnheiten der Willkürschreiber}

Die Entstehungsdaten von den der Sprachanalyse unterzogenen Schriftstücken lassen vermuten, dass hier der Entwicklungsstand des Deutschen aus der (früh-) neuhochdeutschen (fnhd.) Periode erforscht wird, wobei mitberücksichtigt werden muss, dass die fnhd. Quantitätsverschiebungen nur als Glied in der langen Kette der deutschen Sprachgeschichte zu betrachten sind. ${ }^{5}$

Die Langvokale, deren graphische Fixierung in den Fokus dieses Beitrags gestellt wurde, sind aber das Resultat ihres Dehnungsprozesses in den offenen Tonsilben, der ab dem 12. Jh. im Nordwesten des deutschsprachigen Gebietes merklich ist, von wo aus er sich wellenweise verbreitete, um schließlich in den ganzen mittel- und oberdeutschen Raum zu gelangen (vgl. Szulc 1969:71, Mettke 1970:69-70, Schmidt 1980:289-290, Morciniec 2015:126-127). Der entgegengesetzte Prozess, der seit dem Ende des 12. Jh. in manchen mitteldeutschen Mundarten gesichtet werden kann, ist die Vokalkürzung in den geschlossenen Silben (vgl. Szulc 1969:71, Mettke 1970:71-72, Schmidt 1980:291, Morciniec 2015:127-128), während deren die mhd. langen Vokale gekürzt wurden. Wie aber schon oben festgestellt wurde, geht die Schwierigkeit der Ermittlung der fnhd. Schreibung aus der fehlenden allgemeingültigen Konvention einer konsequen-

${ }^{5}$ Die Quantitätsopposition: Langvokal : Kurzvokal war schon im (Ur-)germanischen (germ.) präsent und beim Übergang zum Althochdeutschen (Ahd.) wurde die Entstehung der langen Vokale infolge der Verschmelzung von germ. Diphthongen festgestellt: germ. [ai] > ahd. [e:] und germ. [au] $>$ ahd. [o:], z.B. got. saihwan $>$ ahd. sēula, sēla $>$ mhd. sēla $>$ nhd. Seele oder got. ausō $>$ ahd. $\bar{r} r \bar{a}>$ mhd. $\bar{o} r e, \bar{o} r>$ nhd. Ohr. Als Konsequenz dieses Lautwandels trat die fuhd. Monophthongierung ein, infolge deren die mhd. Diphthonge zu fnhd. Langvokalen wurden: [ie, uo, ye] > [i:, u:, y:]. 
ten Markierung der Lautdauer hervor. Deswegen lassen sich die graphemischen Prämissen und ihre potenziellen lautlichen Entsprechungen nicht mit absoluter Überzeugung erörtern, d.h. sie sind lediglich Annahmen und sollen nur in einem solchen Licht beobachtet werden (vgl. oben).

Ordnungshalber muss bemerkt werden, dass die Hauptvariante eines jeden Graphems in spitzen Klammern erscheint (z.B. <e>), während ihre Allographen in runde Klammern gesetzt werden [z.B. (eh) oder (ee)]. Die Belege werden zusätzlich hinsichtlich der Entstehungszeit der Willkür gruppiert.

\subsection{Dehnungsprozesse}

Was die Dehnung anbelangt, so muss ausdrücklich festgestellt werden, dass die Schreibung die gedehnten Laute generell äußerst grell und deutlich markiert, obwohl auch manche graphischen Inkonsequenzen aufgezeigt wurden, die aber auf dem Wege der Analogie im Verhältnis zu anderen Belegen annehmen lassen, dass der Laut der Dehnung unterlag.

fnhd. [a:] $(<$ mhd. [a]):

17. Jh.

$<\mathrm{a}>$ :

aber, haben, habenn, Vater, Gevattern, Tage, Sontage, Feyertage, Feyertagen, Tages, Tagk, Tagen, untersagen, angesaget, Scharwercke, Scharwergk, Scharwercks, Schawerck, scharwercken, Klage, Klagen, Laden, Vertrag, tragen, antragen, Schaden, Schade, Schadens, Lagerholcz, Graben, beschlagen, iagen, Laden;

(ah): $(-\mathrm{V}+<\mathrm{h}>-)$ : Wahren, Nahmen, fahren, Nahrung, bewahren, bezahlenn, bezahlet, zahlen, zahlenn.

Im Falle von Taffel ist die fehlende Schreibungskonsequenz augenfällig, die darin besteht, dass die verdoppelte graphische Variante des konsonantischen Lautes auf das vokalische graphische Zeichen folgt $\left(-\mathrm{V}+\mathrm{K}_{1}+\mathrm{K}_{1^{-}}\right)$(vgl. unten).

18. Jh.

$<\mathrm{a}>$ :

(aа): $\left(-\mathrm{V}_{1}+\mathrm{V}_{1^{-}}\right)$: maaß; aber, abermahln, schade, schaden, schadens, schadleidenden, schadhafft, ansagen, angesagten, anzusagen, beklagte, tag, tage, tages, taglöhner, sonn- undt feier tagen, namentlich, maße, graben, gegrabenen, waßergraben, mag; 
(ah): $(-\mathrm{V}+<\mathrm{h}>-): \quad$ zahlen, gezahlet, zahl, verfahren, überfahrung, fahren, abermahln, andermahl, andermahln, mahl, niemahl $\beta$, verwahre.

fnhd. $[\varepsilon:](<\operatorname{mhd} .[\varepsilon])$ :

17. Jh.

$<\mathrm{e}>$ :

dem, den, des, demselbigen, denselben, Predigt, Prediger, denjenigen, abzulegen, ablegenn, ablegen, eingeleget, erlegenn, auflegete, begegnet, abgefeget, hierkegen, geheget, gehegte, gehegten, Hegeweide, Jegendt, daneben, angelegen, entweder, allewege, gebe, nachgegebenn, gegeben, mitzugeben, gewesen, Wege, Stege, pflegen, Reden, vorgelesen, leben;

$<\mathrm{e}>$ : $\quad$ Dehnung vor $/ \mathrm{r} / \mathrm{auch}$ in geschlossenen Silben im gesamten hochdeutschen Gebiet: der, derselbigenn, derselben, Pferdearbeit, Pferdt, Pferde, werden, werdenn, werde;

(ä):

Kirchenväter, Kirchenvätern;

(eh): $(-\mathrm{V}+<\mathrm{h}>-)$ : $\quad$ befehlen, versehen, zusehenn, ansehe, besehet, ersehen, Befehlich, aufnehmen;

(eeh): $\left(-\mathrm{V}_{1}+\mathrm{V}_{1}+<\mathrm{h}>-\right)$ : seehenn.

18. Jh.

$<\mathrm{e}>$ :

ebenmäßig, ebenmeßig, ebener maßen, erwegung, der, dem, demselben, dem selben, den, erbettener, gegen, nemlich, allewege, gelegen, ungelegenheit, gelegenheit, ablegen, abzulegen, zugelegener, zuerlegen, dafernenebst, nebenst, erheben, besheret, anwesenden, abgeben, zubegeben, außgegeben, gegeben, übergeben;

$<\mathrm{e}>$ : $\quad$ Dehnung vor $/ \mathrm{r} /$ auch in geschlossenen Silben im gesamten hochdeutschen Gebiet: er, erde, zubeerdigen, dero, derselben, derselbe, der jenige, pferde, pferden, pferdt;

(ä): schlägen, nämlich;

(eh/ah): $(-\mathrm{V}+<\mathrm{h}>-)$ : erwahlung, erwehlung, zuerwahlen, erwehlet, dehnen ,denen', Nachdehm, erbehten, geschehen, nehmlich, zuversehen, versehe, wegnehme, einzunehmen, zunehmen, ablehnete, stehlen, begehret.

Bei erbettener ist die Doppelkonsonanz augenfällig, die aber keine Kürze des vorangehenden Vokals signalisiert. 
fnhd. [i:] $(<$ mhd. [I]):

17. Jh.

$<\mathrm{i}>$ :

(ih):

Dehnung vor $/ \mathrm{r} /$ auch in geschlossenen Silben im gesamten hochdeutschen Gebiet: ihrer, ihre, ihr, ihren, wir;

(ie): $(<-\mathrm{i}+\mathrm{e}->$ : $\quad$ viel, vielweniger, liegen, wiederumb, dawieder, Spielen, mitgespielet, auffetrieben, Wiesen, aussbliebe, verwiesen, verschrieben;

(ieh): $(<-\mathrm{i}+$ eh->: $\quad$ geschiehet, siehet, Vieh, Viehe;

(iech): $(<-\mathrm{i}+$ ech- $>$ : geschiecht;

(e):

Dorffrede.

Im Falle von (ie) und seinen anderen oben präsentierten Alternanten muss aber darauf aufmerksam gemacht werden, dass dieselbe Schreibung auch bei der schriftlichen Fixierung des fnhd. [i:] aufgezeigt wird, das infolge der Monophthongierung des mhd. Diphthongs [ie] entstand (mhd. [ie] $>$ fnhd. [i:]). Gerade dieses aus dem alten Diphthong stammende $<\mathrm{e}>$ wurde zum orthographischen Zeichen, das auf andere Wörter mit dem gedehnten [I] (= [i:]) auf dem Wege der Analogie - auch in solchen Fällen, wo das $<\mathrm{e}>$ etymologisch nicht motiviert ist - übertragen wurde: (17. Jh.): lieben, Lieb, Beliebung, Dienstbothen, Dienern, Hoffdienste, Verfliessung, verbieten, gebieten, Niemandt, Kirchendienern, Bier, Biers, hierkegen, abzuschiessen, schiessen, Verliehrung, Miethe, (18. Jh.): gebietende, liebe, hierin, hierinnen, bier, fließen, tieff, zugebieten, vierte, vierthe, ließe, genießen, diebe, hiemitt, hiemit, niemahlß

18. Jh.

$<\mathrm{i}>$ :

irer, wir;

(ih):

ihrer, ihrem, ihnen, ihm, ihn;

(ie): $(<-\mathrm{i}+\mathrm{e}->$ :

Wier, zufrieden, verschwiegenheit, vieler, viellweniger, viell, viellmehr, imwiedrigem, wiedrigen, diesem, diese, gliedes, dawieder, wieder rede, getrieben, lieget;

(ieh): $(<-\mathrm{i}+$ eh- $>$ : $\quad$ geliehen, vieh, viehes, vieh-pfendung.

Im Falle von viellweniger, viell, viellmehr wird die Doppelkonsonanz vorgefunden, die aber mit der Kürze des vorangehenden Vokals nichts zu tun hat. Außerdem ist Schreibungsinkonsequenz sichtbar: vieler vs. viellweniger, viell, viellmehr.

fnhd. [u:] (< mhd. [v]):

17. Jh.

$<\mathrm{u}>$ : $\quad$ Ursach;

(uh): $(-\mathrm{V}+<\mathrm{h}>-)$ : $\quad$ uhralten; 
18. Jh.

$<\mathrm{u}>$ :

(uh): $(-\mathrm{V}+<\mathrm{h}>-)$ : uhrsachen.

fnhd. [y:] $(<\operatorname{mhd}$. [Y] $)$ :

17. Jh.

$<\ddot{\mathrm{u}}>$ : $\quad$ darüber, für, fürfalle;

(üh): $(-\mathrm{V}+<\mathrm{h}>-)$ : gebührende, gebührlicher, gebührlichen, Gebühr, Mühlen;

(u) ${ }^{6}: \quad$ uber, uberspringen, Ubelthat;

18. Jh.

$<\ddot{\mathrm{u}}>$ :

für, fürs, überreichte, übell, darüber, übergeben, thüren;

(üh): $(-\mathrm{V}+<\mathrm{h}>-)$ : willkühr, willkührliche, willkührlicher, willkührlichen, hinführo, gebührende.

fnhd. [o:] $(<\operatorname{mhd} .[0])$ :

17. Jh.

$<_{0}>$ :

osterliche $^{7}$, Oster-, oder, Obrigkeit, Backofen, oben, Geboten, Dienstbothen, Gastgeboten, verbotene, verboten, Gewonheit, sowol, Vorwercks, holen, vorbehalten, vorgelesen, Zobeln, Vorwissen, Vorjahr, wonhafftig, Vogel.

Im Falle von Botten, verbotten, Verbott, sowoll, Boddem, Hoffdienste, Hoffe, Hoff, woll lässt sich orthographische Inkonsequenz feststellen, weil der verdoppelte graphische Alternant des konsonantischen Graphems auf das vokalische graphische Zeichen folgt $\left(-\mathrm{V}+\mathrm{K}_{1}+\mathrm{K}_{1^{-}}\right)$(vgl. oben).

18. Jh.

$<_{0}>$ :

vorgenommene, vorm, vorbehalt, vors, vorgestehener, vormundtschafft, vorhin, hoffe, hoff, kirchhoff;

(oh): $(-\mathrm{V}+<\mathrm{h}>-): \quad$ gewohnet, wohnen, erkohren, befohlen, gestohlen.

fnhd. [ø:] $(<\operatorname{mhd} .[œ])$ :

17. Jh.

\footnotetext{
${ }^{6}$ Unbezeichneter Umlaut.

7 Vgl. österlich, adj., ahd. ôstarlîh, mhd. osterlîch und auch im älteren nhd. noch oft ohne Umlaut, (vgl. Deutsches Wörterbuch von Jacob und Wilhelm Grimm. 16 Bde. in 32 Teilbänden. Leipzig 1854-1961. Quellenverzeichnis Leipzig 1971. Online-Version: http:/woerterbuchnetz.de/cgi-bin/ WBNetz/wbgui_py?sigle=DWB\&mode $=$ Vernetzung\&lemid $=$ GO02519\#XGO02519, 10.03.2018).
} 
$<$ ö>: $\quad$ mögen, vermöge $e^{8}$, gewönlichem, Vögel;

(öh): $(-\mathrm{V}+<\mathrm{h}>-):$ Söhnen ${ }^{9}$;

18. Jh.

$<\ddot{0}>$ :

möge, vermöge, vermögen, vermöge, vermögen ${ }^{10}$;

zutrösten, gewöhnliche.

\subsection{Kürzungsprozesse}

Im Falle der Kürzung muss die Analyse sehr vorsichtig vorgenommen werden, weil die graphischen Äquivalente der Laute nicht immer konsequent gebraucht werden, was zu der Feststellung verleiten könnte, dass der Laut nicht gekürzt worden sei. Die Doppelkonsonanz nach einem Vokal $\left(-\mathrm{V}+\mathrm{K}_{1^{-}}+\mathrm{K}_{1^{-}}\right)$kann auch stellenweise irreführend sein (vgl. oben: Taffel).

fnhd. [a] (< mhd. [a:]):

17. Jh.

$<\mathrm{a}>$ : Andacht;

$<\mathrm{a}>\left(-\mathrm{V}+\mathrm{K}_{1}+\mathrm{K}_{1^{-}}\right)$: lassen, lassenn, Zulass, einlassen, erlassen;

18. Jh.

$<\mathrm{a}>$ : andacht;

$<\mathrm{a}>\left(-\mathrm{V}+\mathrm{K}_{1}+\mathrm{K}_{1^{-}}\right)$: laßen, außlaßen, zulaßen, zugelaßen;

fnhd. $[\varepsilon](<\operatorname{mhd}$. [e:]):

17. Jh.

$<\mathrm{e}>\left(-\mathrm{V}+\mathrm{K}_{1}+\mathrm{K}_{1^{-}}\right)$: Herrschaft, Herrschafften, Nachlessigkeiten, nachlessig, nachlessigk;

18. Jh.

$<\mathrm{e}>\left(-\mathrm{V}+\mathrm{K}_{1}+\mathrm{K}_{1-}\right)$ : herrschafft;

(ä): (-V $\left.+\mathrm{K}_{1}+\mathrm{K}_{1-}\right)$ : lässet, läßet, unnachläßlich.

fnhd. [I] $(<$ mhd. [i:] < mhd. [ie]) $(<$ ahd. /ie/ $</$ io/ $</$ eo $/)($ nach Kürzung des Diphthongs vor/-cht/, vgl. Kluge 2011:576):

17. Jh.

$<\mathrm{i}>\left(-\mathrm{V}+\mathrm{K}_{1}+\mathrm{K}_{2}-\right)$ : Fichten, Lichten, Lichte;

8 Nach Senkung und darauffolgender Dehnung.

9 Nach Senkung und darauffolgender Dehnung.

10 Nach Senkung und darauffolgender Dehnung. 
18. Jh.

$<\mathrm{i}>\left(-\mathrm{V}+\mathrm{K}_{1}+\mathrm{K}_{2}-\right):$ Lichtmeß;

$<\ddot{u}>=[\mathrm{y}](<[\mathrm{y}:]<[\mathrm{ye}])$ (nach Monophthongierung und darauffolgender Kürzung): unverbrüglich.

fnhd. [0] $(<$ mhd. [o:]):

17. Jh.

$<_{0}>(-\mathrm{V}+[\mathrm{x}]-): \quad$ Hochzeiten $($ vgl. oben $)$.

\section{Abschließendes}

Die Sprachanalyse der Schriftstücke aus dem 17. und 18. Jh. bewies eindeutig, dass die Grapheme, die die infolge der fnhd. Dehnung bzw. Kürzung entstandenen Laute graphisch darstellen, sehr viele Alternanten besitzen, wobei ihre Gebrauchskonsequenz nicht zu bestimmen ist, d.h. in den konkreten Belegen kommen unterschiedliche Allographe desselben Graphems vor - sogar in denselben Wörtern oder in den verschiedenen Formen desselben Wortes - z.B.

- versehen, zusehenn, ansehe, besehet, ersehen: seehenn (17. Jh.);

- irer : ihrer (18. Jh.);

- wir: Wier (18. Jh.);

- dem, demselben: Nachdehm (18. Jh.);

- erbettener : erbehten (18. Jh.);

- nemlich : nämlich : nehmlich (18. Jh.);

- vieler : viellweniger, viell, viellmehr (18. Jh.).

Dieser Variantenreichtum, der eher für das Dokument aus dem 18. Jh. kennzeichnend ist, zeugt von den zahlreichen mühsamen Versuchen seitens des Schreibers (bzw. der Schreiber), die dezentesten Eigenheiten der lebendigen Rede, der die Vokallänge und -kürze zugerechnet werden, möglichst genau visuell darzustellen. Die Alternanz bestimmter Lösungen lässt wiederum den Schluss zu, dass der Schreibende ständig auf der Suche nach der möglichst besten Option für die schriftliche Fixierung eines gegebenen Lautes war.

Darüber hinaus wird die fehlende Konsequenz der Schreibung festgestellt, die sich im Gebrauch mindestens zweier Buchstaben nach dem den vokalischen Laut schriftlich fixierenden Graphem äußert: Taffel (17. Jh.), Botten, verbotten, Verbott, sowoll, Boddem, Hoffdienste, Hoffe, Hoff, woll (17. Jh.), erbettener (18. Jh.), viellweniger, viell, viellmehr (18. Jh.), wo Doppelkonsonanz sichtbar ist, mit der aber keine Kürze des vorangehenden Vokals zum Ausdruck gebracht wird.

Die Opposition von Belegen: nachlessig, nachlessigk (17. Jh.): lässet, läßet, unnachläßlich (18. Jh.) veranschaulicht hingegen die Veränderung der Lautnie- 
derschreibung sowie den Versuch des Gebrauchs einer Graphemvariante, die das morphologische Paradigma des Lautes notiert und auf die Etymologie des Lautes hinweist.

Die obigen Erwägungen über die breite Palette der Allographe, die in der Niederschreibung der Ergebnisse der fnhd. Dehnung und Kürzung merklich sind, lassen also die Konstatierung von Siegfried Grosse (1924-2016) zitieren, der behauptet, dass ,[...] die Schrift ein sekundär entwickeltes artifizielles System [ist], um das Gesprochene optisch zu fixieren und jederzeit verfügbar zu machen“ (Grosse 1999:205).

\section{Quellen}

Kutrzeba Stanisław / MaŃKowski Alfons, 1938, Wilkierz dla wsi klasztoru Panny Marji w Kartuzach. In: Archiwum Komisji Prawniczej, Tom XI, Warszawa/Kraków/Lódź/Poznań/Wilno/Zakopane, S. 23-31, http://dir.icm.edu.pl/Archiwum_Komisji_Prawniczej/Tom_11/ (20.02.2018).

TARGOwski Michał (Hrsg,), 2013, Wilkierz wsi Kosowo i Chrystkowo z pierwszej połowy XVIII wieku, Gruczno.

\section{Sekundärliteratur}

Baudouin de Courtenay Jan, 1974, Dzieła wybrane, Warszawa.

BLOOMFIELD Leonard, 1933, Language, London.

Снвомік Grzegorz, 2010, Schreibung und Politik. Untersuchungen zur Graphemik der frühneuhochdeutschen Kanzleisprache des Herzogtums Teschen, Kraków.

GrabareK Józef, 2004, Die Ostkolonisation im westslawischen und baltischen Sprachraum bis 1350, in: Bartoszewicz I./Hałub M./Jurasz A. (Hrsg.), Werte und Wertungen. Sprach-, Literatur- und kulturwissenschaftliche Skizzen und Stellungnahmen. Festschrift für Eugeniusz Tomiczek zum 60. Geburtstag, Wrocław, S. 504-512.

Grimm Jacob / Grimm Wilhelm, 1971, Deutsches Wörterbuch. 16 Bde. in 32 Teilbänden, Leipzig. Online-Version: http://woerterbuchnetz.de/cgi-bin/WBNetz/wbgui_py?sigle=DWB (10.03.2018).

Grosse Siegfried, 1999, Sprechen und Schreiben, in: Vielfalt der Sprachen. Festschrift für Aleksander Szulc zum 75. Geburtstag, Wien.

Höchli Stefan, 1981, Zur Geschichte der Interpunktion im Deutschen. Eine kritische Darstellung der Lehrschriften von der zweiten Hälfte des 15. Jahrhunderts bis zum Ende des 18. Jahrhunderts, Berlin/New York.

KLUGE Friedrich, 2011, Etymologisches Wörterbuch der deutschen Sprache, Berlin/Boston.

Metтke Heinz, 1970, Mittelhochdeutsche Grammatik, Leipzig.

MoRCinIEC Norbert, 2015, Historia języka niemieckiego, Wrocław.

OwsiŃSKI Piotr A., 2017, Graphematische Untersuchungen zur ostdeutschen Apostelgeschichte aus dem 14. Jahrhundert, Frankfurt am Main.

Paul Hermann, 1968, Prinzipien der Sprachgeschichte, Tübingen.

Paveau Marie-Anne / Sarfati Georges-Élia, 2009, Les grandes théories de la linguistique. De la grammaire comparée à la pragmatique (tłum. Piechnik I., Wielkie teorie językoznawcze. Od językoznawstwa historyczno-porównawczego do pragmatyki), Kraków. 
PentTILÄ Aarni, 2011, Zur Grundlagenforschung der geschriebenen Sprache, in: Acta Universitatis Upsaliensis. Acta Societatis Linguisticae Upsaliensis 2:2 1970, http://www.kaapeli.fi/aarnipenttila/ActaUniversitatis.htm (30.10.2011).

Schmidt Wilhelm, 1980, Die Geschichte der deutschen Sprache. Mit Texten und Übersetzungshilfen, Berlin.

SzczepaniaK Renata, 2007, Der phonologisch-typologische Wandel des Deutschen von einer Silben- zu einer Wortsprache, Berlin.

Szulc Aleksander, 1969, Abriss der diachronischen deutschen Grammatik. Teil I. Das Lautsystem, Warszawa/Halle (Saale).

Szulc Aleksander, 1987, Historische Phonologie des Deutschen, Tübingen.

TARGowski Michał, 2013, Wstęp, in: Targowski M. (Hrsg.), Wilkierz wsi Kosowo i Chrystkowo z pierwszej połowy XVIII wieku, Gruczno, S. 7-16.

UlDALL Hans Jørgen, 1944, Speech and writing, in: Acta Linguistica 4, S. 11-16.

\section{The marking of the vowel length in selected village charters from the $17^{\text {th }}$ and $18^{\text {th }}$ centuries. A graphemic-phonemic study}

The paper presents the results of the language analysis of the (Early) New High German village charters from the $17^{\text {th }}$ and $18^{\text {th }}$ centuries which come from: Archiwum Komisji Prawniczej, Volume XI, (Warszawa/Kraków/Łódź/Poznań/Wilno/Zakopane, 1938) and Targowski (2013). The scriveners are unknown. The center of attention are the ways of marking of the long and short vowels, which came into being owing to the lengthening and shortening of the vowels in the Early New High German time. The aim of the article is to determine to what extent the script fixes the features of the spoken language. The author introduces the results of his analysis, illustrating the characteristic features with appropriate examples.

Keywords: lengthening, shortening, vowel, village charter, New High German. 